\title{
COVID19 Classification Using CT Images via Ensembles of Deep Learning Models
}

\author{
Abdul Majid ${ }^{1}$, Muhammad Attique Khan ${ }^{1}$, Yunyoung Nam ${ }^{2, *}$, Usman Tariq ${ }^{3}$, Sudipta Roy ${ }^{4}$, \\ Reham R. Mostafa ${ }^{5}$ and Rasha H. Sakr ${ }^{6}$
}

\author{
${ }^{1}$ Department of Computer Science, HITEC University Taxila, Taxila, 47080, Pakistan \\ ${ }^{2}$ Department of Computer Science and Engineering, Soonchunhyang University, Asan, Korea \\ ${ }^{3}$ College of Computer Engineering and Sciences, Prince Sattam Bin Abdulaziz University, Al-Khraj, Saudi Arabia \\ ${ }^{4}$ PRTTL, Washington University in Saint Louis, Saint Louis, MO 63110, USA \\ ${ }^{5}$ Department of Information Systems, Faculty of Computers and Information Sciences, Mansoura University, \\ Mansoura, 35516, Egypt \\ ${ }^{6}$ Department of Computer Science, Faculty of Computers and Information Sciences, Mansoura University, \\ Mansoura, 35516, Egypt \\ *Corresponding Author: Yunyoung Nam. Email: ynam@sch.ac.kr \\ Received: 12 January 2021; Accepted: 20 March 2021
}

\begin{abstract}
The recent COVID-19 pandemic caused by the novel coronavirus, severe acute respiratory syndrome coronavirus 2 (SARS-CoV-2), has had a significant impact on human life and the economy around the world. A reverse transcription polymerase chain reaction (RT-PCR) test is used to screen for this disease, but its low sensitivity means that it is not sufficient for early detection and treatment. As RT-PCR is a time-consuming procedure, there is interest in the introduction of automated techniques for diagnosis. Deep learning has a key role to play in the field of medical imaging. The most important issue in this area is the choice of key features. Here, we propose a set of deep learning features based on a system for automated classification of computed tomography (CT) images to identify COVID-19. Initially, this method was used to prepare a database of three classes: Pneumonia, COVID19, and Healthy. The dataset consisted of 6000 CT images refined by a hybrid contrast stretching approach. In the next step, two advanced deep learning models (ResNet50 and DarkNet53) were fine-tuned and trained through transfer learning. The features were extracted from the second last feature layer of both models and further optimized using a hybrid optimization approach. For each deep model, the Rao-1 algorithm and the PSO algorithm were combined in the hybrid approach. Later, the selected features were merged using the new minimum parallel distance non-redundant (PMDNR) approach. The final fused vector was finally classified using the extreme machine classifier. The experimental process was carried out on a set of prepared data with an overall accuracy of $95.6 \%$. Comparing the different classification algorithms at the
\end{abstract}

This work is licensed under a Creative Commons Attribution 4.0 International License, which permits unrestricted use, distribution, and reproduction in any medium, provided the original work is properly cited. 
different levels of the features demonstrated the reliability of the proposed framework.

Keywords: COVID19; preprocessing; deep learning; information fusion; firefly algorithm; extreme learning machine

\section{Introduction}

Coronavirus Disease 2019 (COVID-19) is a contagious disease caused by the novel coronavirus, severe acute respiratory syndrome coronavirus 2 (SARS-CoV-2) [1], which was first recognized in Wuhan, China, at the end of 2019 [2]. The COVID-19 pandemic has had a major effect on public health around the world. The symptoms of COVID-19 include fever, headache, shortness of breath, muscle pain, fatigue, and sore throat [3]. Severe outcomes of this disease include organ failure, severe respiratory problems, and death. Approximately 20\% of COVID-19 cases become severe, and it has an estimated mortality rate of 3\% [4]. This disease is detected by a reverse transcription polymerase chain reaction (RT-PCR) test, but as the RT-PCR test has low sensitivity it is not sufficient for early detection and treatment [5]. As RT-PCR is a time-consuming procedure, the application of artificial intelligence (AI) and deep learning-based systems would save a great deal of time for physicians [6]. As of February 2021, the total number of recorded COVID-19 cases worldwide is 106,440,335, with 2,322,351 deaths. These statistics show that approximately $3.4 \%$ of deaths around the entire world are due to COVID-19.

Deep learning has been successfully applied in medical imaging [7-9]. The major areas where medical imaging is applied are in the detection of skin cancer [10], brain tumors [11], stomach cancer [12], lung cancer [13], breast cancer [14], blood cancer [15], and COVID-19 [16,17]. Several techniques have been introduced by computer vision (CV) researchers for early coronavirus recognition using chest X-ray [18] and computed tomography (CT) [19] images, and AI and deep learning models have been applied for accurate classification [20,21]. A system has been designed for prediction and detection of COVID-19 on chest X-rays using deep learning [22]. This method is based on $\mathrm{AI}$ and uses the features of a convolution neural network (CNN). In the detection phase, three algorithms are used, i.e., the Autoregressive Integrated Moving Average (ARIMA) method, the Prophet Algorithm (PA), and the Long Short-Term Memory (LSTM) neural network. Many other methods are also discussed in the Related Work section.

Several issues remain that are related to the classification of COVID-19 using CT images. The first is the availability of high-dimensional datasets, as an extensive dataset is always needed to train a deep learning model. The second is the selection of a deep model for feature extraction. Several deep models are available for classification purposes and, therefore, the most useful model must be chosen. The third and most important issue is the existence of redundant and irrelevant features that increase the computational time and affect the accuracy of classification. However, some relevant features that are important for improving accuracy are sometimes discarded during the selection of important features.

Here, we propose a new method based on a set of deep learning models. The main purpose of this work was to allow the selection of optimal features from two pre-trained deep learning models and then combine their information. As mentioned above, important features may also be lost in the selection stage. Therefore, optimal information from the two models is used to fill the gap, which improves the accuracy of classification. In this work, we prepared a database of three classes: Pneumonia, COVID-19, and Healthy. The prepared dataset consisted of a total of 6000 CT images adjusted with two pre-trained deep learning models (ResNet50 and DarkNet53) and 
trained by transfer learning. Later, the features were extracted from the second last layer called the feature layer. A hybrid optimization method is proposed based on the hybrid Rao-1 algorithm and the PSO algorithm. The features of both deep models were optimized by this hybrid method for the next step. Optimal features were merged using a new approach called Parallel Minimum Distance Non-Redundant (PMDNR).

The rest of the article is organized as follows. Related work is discussed in Section 2. Section 3 presents the proposed methodology with the detailed mathematical formulation. The results and discussion are presented in Section 4. Section 5 presents the conclusion.

\section{Related Work}

In 2020, image processing and CV researchers worked to develop systems for diagnosing and recognizing COVID-19 on CT and X-ray images. These detection systems focused mainly on deep learning to improve efficiency and performance. Apostolopoulos et al. [23] introduced a deep learning-based approach for recognizing COVID-19 on X-ray images. In this method, transfer learning was performed using state-of-the-art CNN models, including VGG-19, Inception ResNet v2, Inception, Xception, and MobileNet v2. The best results were obtained with MobileNet v2, with specificity, accuracy, and sensitivity of $96.46 \%, 96.78 \%$, and $98.66 \%$, respectively. Ozturk et al. [24] developed a CNN-based automated system for recognizing coronavirus in chest X-ray images, which implemented You Only Look Once (YOLO) and used the DarkNet network for classification to achieve a binary class recognition rate of $98.08 \%$. Ismael et al. [25] presented a CNN approach to recognizing COVID-19 in chest X-ray images. In this approach, different deep learning models were used for feature extraction. The extracted features were then passed to a disease-recognition support vector machine (SVM). The maximum accuracy achieved by this method was 94.7\%. Panwar et al. [26] developed a CNN-based technique for the rapid detection of COVID-19 on chest CT and X-ray images. The transfer learning approach was implemented on three different datasets. This technique detected COVID-19 in less than 2 min and produced more accurate results from CT images than the X-ray dataset. A new CNN framework called CoroDet [27] was introduced to recognize COVID-19 on chest X-ray and CT images. The network consisted of 22 layers, and had a recognition rate of $99.1 \%$ for binary class classification, $94.2 \%$ for three classes, and 91.2\% for four classes. Islam et al. [28] presented a CNN-based method for the detection of COVID-19. In this method, the CNN extracted features that were then used to classify the dataset into normal and COVID-19 images using LSTM. This technique achieved an area under the receiver operating characteristic (ROC) curve (AUC) of 99.9\%, accuracy of 99.4\%, sensitivity of $99.3 \%$, specificity of $99.2 \%$, and F1 score of $98.9 \%$.

A new artificial neural network (ANN), CapsNet [29], was introduced to detect COVID19 in a chest X-ray image dataset. The network showed recognition accuracies for binary and multi-class classification of $97.24 \%$ and $84.22 \%$, respectively. A dual-branch combination network (DCN) [30] was established to detect and diagnose COVID-19 on chest CT. The lesion area was first segmented to obtain more accurate results in the classification phase, and the DCN model achieved an accuracy of $96.74 \%$. Horry et al. [31] used the transfer learning approach to diagnose COVID-19 in ultrasound, X-ray, and CT images. They selected the VGG-19 network to perform transfer learning and made appropriate changes to the parameters to fine-tune the model. The technique showed accuracies of $100 \%, 86 \%$, and $84 \%$ for ultrasound, X-ray, and CT images, respectively. A number of other methods using statistical modeling [32], a cloud-based framework model [33], and machine learning [34] have also been reported, all of which focused on pre-trained 
models for COVID-19 image classification. However, they focused only on classification accuracy rather than computational time, which is a vital functionality of any computerized method.

\section{Proposed Framework}

The proposed automated set of deep learning methods is presented in this section with the detailed mathematical formulation and visible results. The main flow of the proposed method is shown in Fig. 1. As shown in this figure, the initially collected dataset is processed and the contrast is improved using a hybrid method. Then, two pre-trained models are fine-tuned and trained by transfer learning. Both fine-tuned models are trained in a dataset consisting of three classes: Pneumonia, COVID-19, and Healthy. Features are extracted from these two finetuned models and optimized using a hybrid Rao-1 and PSO algorithm. The selected optimum features are blended using a novel approach called Parallel Minimum Distance Non-Redundant (PMDNR). The final fused vector features are classified using an extreme learning machine (ELM) classifier. The details of each step are presented below.

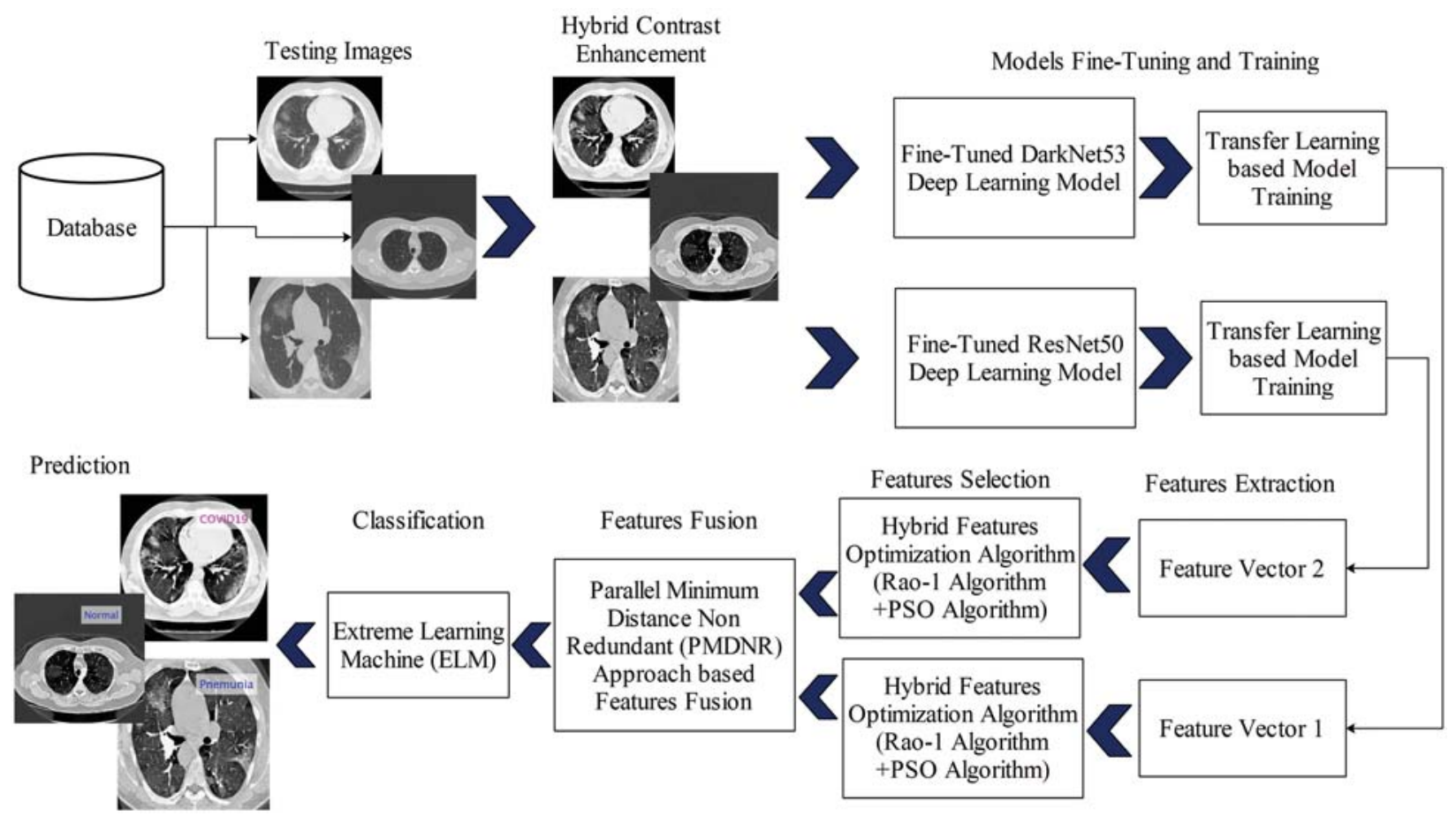

Figure 1: Proposed diagram for COVID19 classification using CT Images based on ensembles of deep learning models

\subsection{Dataset Preparation}

In this work, we collected a dataset from the Radiopaedia Website (https://radiopaedia.org). This website has CT images of more than 100 COVID-19-positive patients. We used the data from the first 80 patients, which included a total of $4000 \mathrm{CT}$ images. Later, we collected $3000 \mathrm{CT}$ images of healthy individuals and $2500 \mathrm{CT}$ images of patients with pneumonia from the same 
website. Due to the imbalance in numbers between the classes, we performed data augmentation with horizontal flip and vertical flip operations. Mathematically, these flip operations are defined as follows:

$O^{H}=I_{i}(n+1-j)$

$O^{V}=I_{(m+1-i)_{j}}$

where $O^{H}$ represents a horizontal flip operation, $O^{V}$ denotes a vertical flip operation, and $I(i, j)$ denotes the original image of dimensions $n \times m$. Based on these operations, all classes were in the range of 4000. Then, we randomly split the entire dataset at a 50:50 ratio for training and testing. A few sample images are shown in Fig. 2. This figure illustrates that the COVID-19 and Pneumonia classes have very high degrees of similarity with each other and, therefore, the chances of misclassification were also very high. To handle these similarity issues, we first implemented a hybrid contrast enhancement approach, as described below.

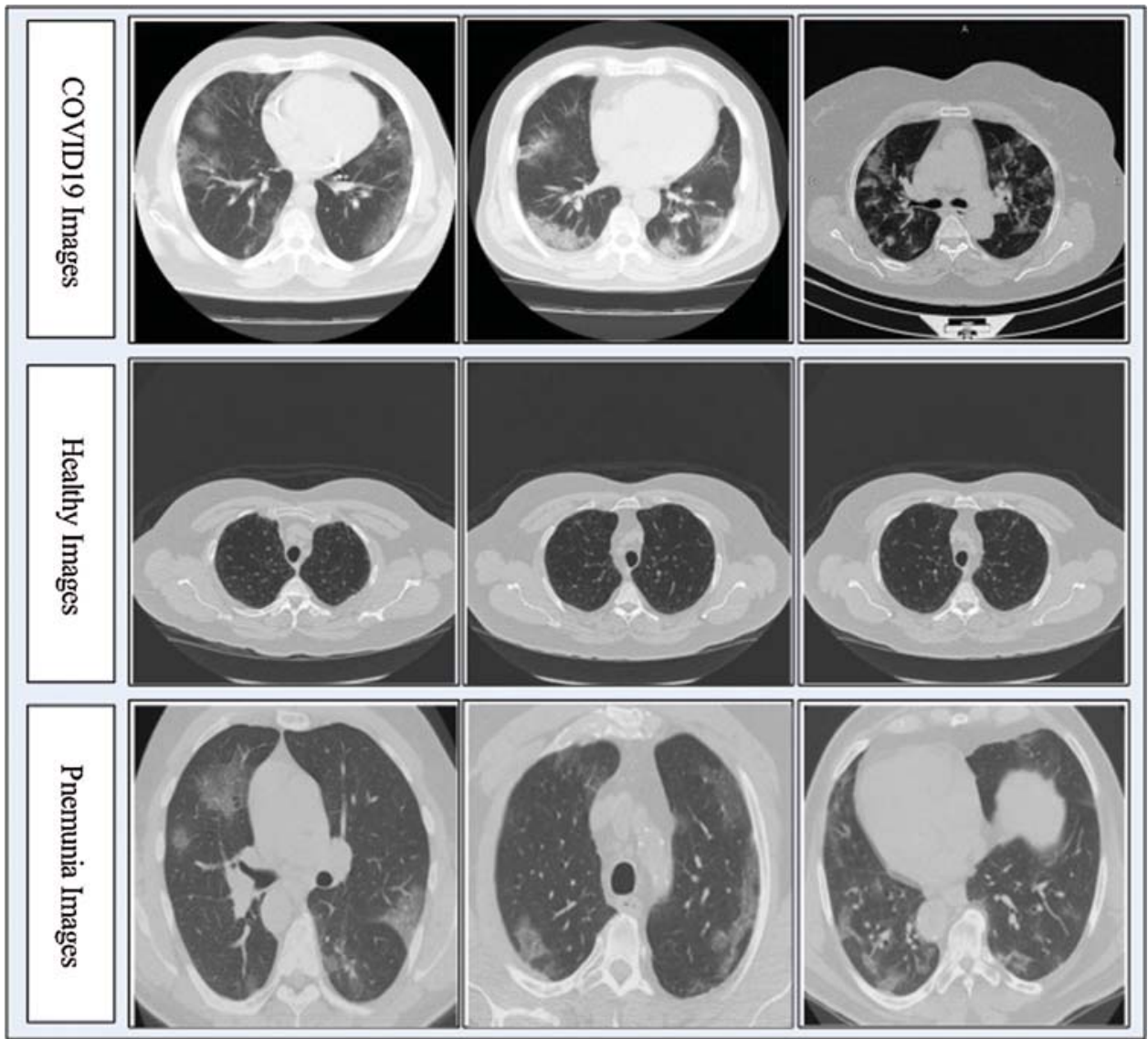

Figure 2: Sample CT images of COVID19, Pneumonia, and Healthy 


\subsection{Contrast Enhancement}

Contrast enhancement improves the quality of input images for useful feature extraction in the pattern recognition step [35]. Here, we implemented a hybrid approach for CT image contrast enhancement. The main purpose of this step was to show a difference between COVID-19 and Pneumonia images. The hybrid approach is based on a new threshold function defined using closing and opening operations, which are applied on original images with a constant parameter. The constant parameter controls the image pixel values and far from zero.

Consider $\Delta$ as a database, $\Delta \in I_{k}^{N}(i, j)$, one image $I(i, j) \in \mathbb{R}$, where the size of the image is $n \times m$, and $n=m=256$. The opening operation is applied on image $I(i, j)$ as follows:

$I_{O p}(i, j)=(I(i, j)-I(i, j)) \circ C+r$

$I_{c l}(i, j)=(I(i, j) . C)-I(i, j)+r$

$I_{f}(i, j)=\left(I_{O p}(i, j)+I_{c l}(i, j)\right)-I(i, j)+r$

$\mu_{1}=\frac{\sum_{i=0}^{m} \sum_{j=0}^{n}\left(I_{O p}(i, j)\right)}{M N}, \mu_{2}=\frac{\sum_{i=0}^{m} \sum_{j=0}^{n}\left(I_{c l}(i, j)\right)}{M N}, \tilde{\mu}=\frac{\mu_{1}+\mu_{2}}{2}$

Thresh $=\left\{\begin{array}{cc}\operatorname{Update}\left(I_{f}(i, j)\right), & \text { for } I_{f}(i, j) \geq \tilde{\mu} \\ I_{f}(i, j), & \text { Elsewhere }\end{array}\right.$

where $I_{O p}(i, j)$ denotes the modified opening function formulation, $I_{c l}(i, j)$ denotes the modified closing operation formulation, $I_{f}(i, j)$ is the combined pixel information formulation, and $\tilde{\mu}$ is the overall mean value. The threshold function Thresh is described as follows: if $I_{f}(i, j)$ image pixels are greater than or equal or $\tilde{\mu}$, then update the pixels' values with the mean value; otherwise, the original pixel is considered. This process is completed for all pixel values, and a new enhanced image is obtained. These steps are illustrated in Fig. 3.

\subsection{Deep Learning Features Extraction}

Deep learning has been an important area of machine learning research for the last 5 years [36]. Many deep learning methods have been introduced for classification purposes, such as object classification [37], medical infections, etc. CNN is a type of deep learning, and consists of many intermediate layers, such as the convolutional layer, pooling layers (max pooling and average pooling), ReLu layer for activation, fully connected layer, and Softmax. The convolutional layer extracts the features of input images based on the filter size. The parameters of this layer are filter size, number of channels, and stride. The pooling layers, such as the max pooling layer, can be useful to handle the issue of overfitting. In this layer, the main parameters are filter size and stride. Usually, the filter size is $3 \times 3$ and stride is 1 . Some of the feature values of the convolutional layers are negative, and it is therefore essential to convert them to positive values. For this purpose, the features are converted into positives through the ReLu activation layer. This layer converted features into zero. The fully connected layer, also called the feature layer, is useful for extracting the features of input images that are transformed through convolutional layers, pooling layers, etc. The final layer is Softmax, used for classification. Here, we used two pre-trained models for deep feature extraction. The details of each model are described below. 


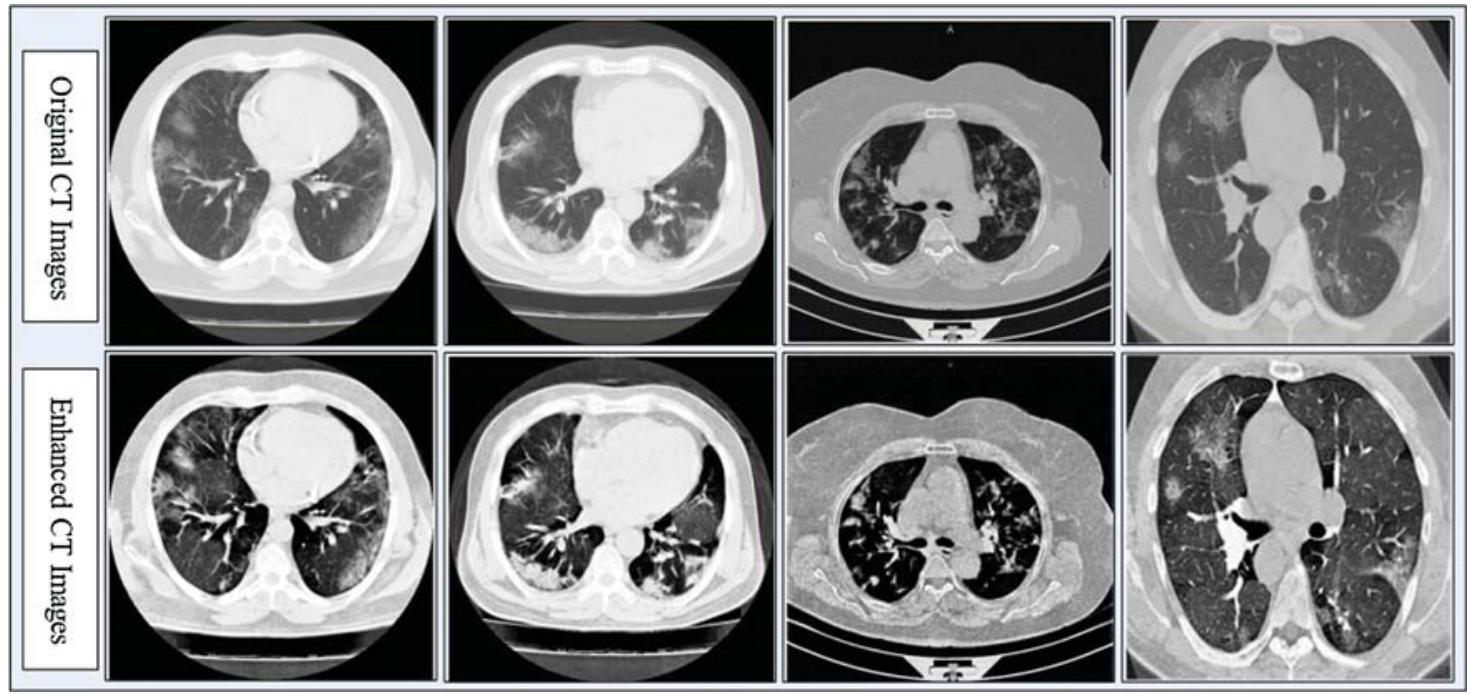

Figure 3: Hybrid contrast enhancement approach results

\subsubsection{The Modified Darknet53 Model}

The CNN-based model DarkNet53 [38] is utilized to extract deep features. Two deep networks named YOLOv2 DarkNet19 [39] and the deep Residual Network [40] were combined to develop this model. The deep structure of DarkNet53 consists of 53 layers. The convolution layer utilized in this network is $1 \times 1$, and the size of the consecutive residual is $3 \times 3$. The input of this network is always $224 \times 224 \times 3$. The minimal component of DarkNet53 consists of three elements: convolution, Batch Normalization (BN), and LeakyReLU layers. The mathematical modeling of the convolutional layer can be described as:

$x_{n}^{k}=\sum_{l \in V_{n}} x_{l}^{k-1} * H_{l n}^{k}+b_{n}^{k}$

where the number of generated feature maps is $n$ by convoluting the input image using $n$ different convolution kernels, and $x_{n}^{k}$ are the $n$ generated feature maps in layer $k$. A feature vector is represented as $V_{n}, H_{l n}^{k}$ represents the $l$ th element of the $n$th number of convolution kernels in layer $k$. $b_{n}^{k}$ is the $n$th bias of the layer $k$, and the convolutional operator is *. The output is normalized using the BN layer. After applying convolution, the BN layer can increase the convergence rate of the network and resolve the problems of overfitting. The mathematical model of the $\mathrm{BN}$ layer is:

$x_{\text {out }}=\frac{\psi\left(x_{\text {conv }}-\mu\right)}{\sqrt{\sigma^{2}}+\varepsilon}+\gamma$

The result of the BN layer is $x_{\text {out }}$. The scaling factor is represented as $\psi$, the mean value of inputs is $\mu$, the input variance value is denoted by $\sigma$, the offset value is $\gamma, \varepsilon$ represents a constant, 
and $x_{\text {conv }}$ denotes the result of the convolution process. LeakyReLU increases the nonlinearity of the network. This is the activation function and can be defined as:

$g_{n}= \begin{cases}x_{n}, & \text { if } x_{n} \geq 0 \\ \frac{x_{n}}{y_{n}}, & \text { if } x_{n}<0\end{cases}$

where $g_{n}$ is the activation function, the input value is $x_{n}$, and $y_{n}$ represents a constant parameter with values between $(1,+\infty)$.

Here, we fine-tuned this model and removed the last classification layer. A new classification layer was added, which included three instead of 1000 classes. The same weights were used for the fine-tuned model. Later, transfer learning was applied to train this model. The transfer learning process is shown in Fig. 4. As shown in the figure, the feature weights of the original model were transferred to the fine-tuned model. The last features were extracted from the convolutional 53 layers of dimension $N \times 1024$.

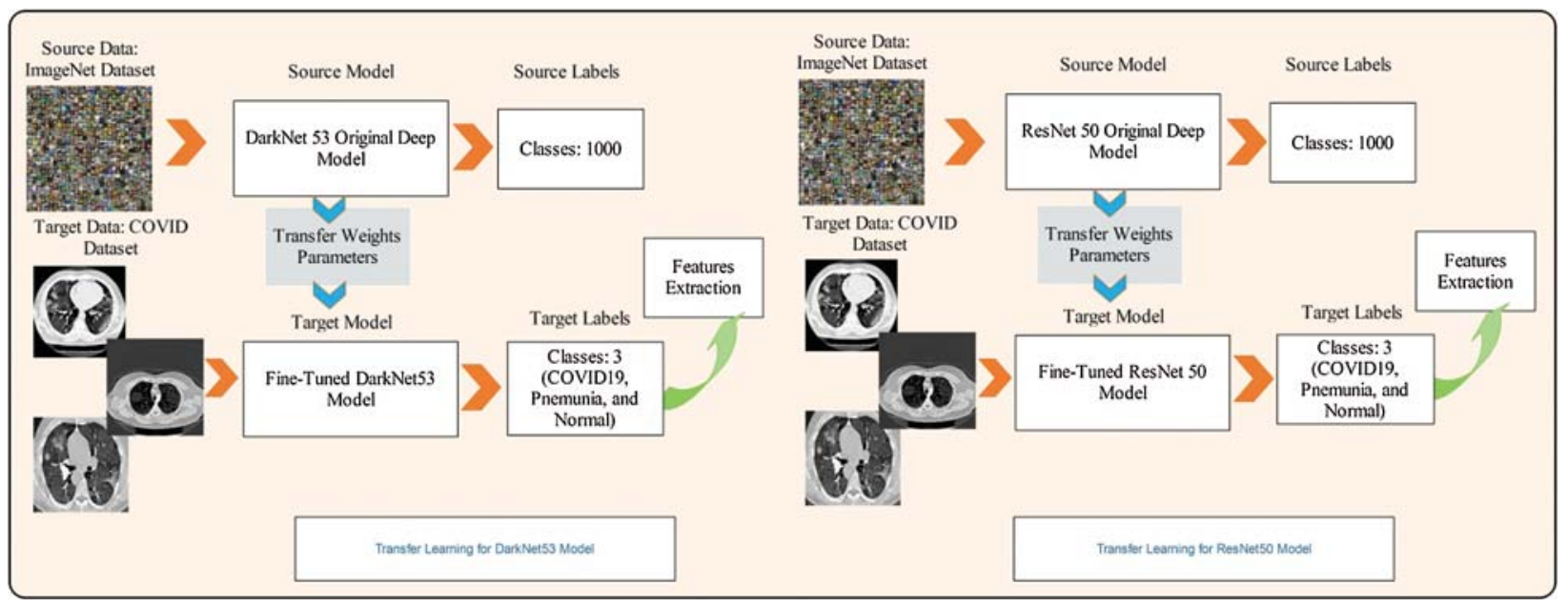

Figure 4: Transfer learning based features extraction

\subsubsection{Modified ResNet50 Model}

ResNet50 [40] is a 50-layer deep neural network based on residual learning that consists of 16 bottleneck residual blocks. The convolution size for each residual block is $1 \times 1,3 \times 3$, and $1 \times 1$. In the first three residual blocks, the feature map sizes are 64 and 256 . The next four blocks have a feature map size of 128 and 512. After this, six residual blocks contain feature maps with sizes of 256 and 1024. The last three blocks have feature map sizes of 512 and 2048. The image input size for ResNet50 is $224 \times 224$, and the feature output size is 2048 . This model was trained on the ImageNet database, a large dataset of millions of images [41]. The output of this original model is 1000 classes, as shown in Fig. 4. We fine-tuned the model and removed the last FC and Softmax layers. A new layer consisting of three classes was added, as shown in Fig. 4. This figure shows that the features weights of the original model were transferred to the fine-tuned model. In the last, features are extracted from the global average pool layer, where the size of extracted features is $N \times 2048$. 


\subsection{Features Selection}

Selection of the best features always helps to achieve faster execution in supervised learning methods. The irrelevant and redundant features mislead the classification accuracy and increase the overall system time [42]. Here, we must deal with both issues of feature redundancy and irrelevant features. Therefore, we proposed a hybrid algorithm based on Rao-1 [43] and PSO [44] to select the most important features. We initially implemented both algorithms separately, identified the correlations among features of both techniques, and selected the most positively correlated features. Finally, the features selected by both approaches were fused using the new PMDNR approach.

Particle Swarm Optimization: This algorithm finds the best possible solution from the given solutions. A swarm is referred to as possible solutions, and each solution is considered a particle. In the search space, each particle set its set and accelerates with some velocity. After each iteration, PSO selected the best solution and changed its position. The new position of the particle is based on previous learning. The evaluation of each particle is measured according to the defined fitness function [45]. At the end of the iterations, PSO produces an optimal solution. Let the search space be $\mathrm{N}$-dimensional with $n$ number of particles or solutions; the $\mathrm{N}$-dimensional vector is denoted by $X_{i}=\left(x_{i 1}, x_{i 2}, \ldots, x_{i N}\right)$ for the $i$ th particle of the swarm. $a_{i}=\left(a_{i 1}, a_{i 2}, \ldots, a_{i N}\right)$ is the prior best position of the $i$ th particle, which results in the best fitness value. $M_{g}$ denotes the particle with the lowest function value. The velocity is represented as $V_{i}=\left(v_{i 1}, v_{i 2}, \ldots, v_{i N}\right)$ for the $i$ th particle. The particles are manipulated according to the following equations:

$v_{i b}^{y+1}=\omega \times v_{i b}^{y}+c_{1} \times \operatorname{rand}() \times\left(a_{i b}^{y}-x_{i b}^{y}\right)+c_{2} \times \operatorname{Rand}() \times\left(a_{g b}^{y}-x_{g b}^{y}\right)$

$x_{i b}=x_{i b}+v_{i b}$

where $b=\{1,2,3, \ldots, N\}, \omega$ is the inertia weight, and random functions are represented by $\operatorname{rand}()$ and $\operatorname{Rand}()$, which generate random values from the range [0,1]. $c_{1}$ and $c_{2}$ are the constant positive integer cognitive, and social parameters, respectively. In the first equation, velocity is calculated at each iteration for the $i$ th particle. $c_{1} \times \operatorname{rand}() \times\left(a_{i b}^{y}-x_{i b}^{y}\right)$ computes the distance between the personal best location and the ith particle. $c_{2} \times \operatorname{Rand}() \times\left(a_{g b}^{y}-x_{g b}^{y}\right)$ gives the distance between the global best location and the $i$ th particle. The second equation calculates the new position of the $i$ th particle. The functions $\operatorname{rand}()$ and $R$ and () provide randomness and make the algorithm more flexible. Through this algorithm, a best-selected feature vector is obtained of dimension $N \times 820$ for feature vector 2 (ResNet features) and $N \times 410$ for feature vector 1 (DarkNet 53 features). This dimension shows that almost $60 \%$ of features are removed from the original list.

Rao-1 Algorithm: This algorithm is computationally complex, easy to implement, and to the point. This algorithm solves the optimization problem more efficiently than other metaheuristic algorithms. The search process for finding an optimal solution is based on the best and worst candidate solutions within a random and entire population.

This algorithm first initializes the parameters, such as the number of solutions, design variables, lower and upper bounds, and maximum iterations. Then, the candidate solutions are generated and a fitness function is applied for evaluation. Initially, the fitness function counter 
and interactions are zero. Based on the fitness values, the best and worst feature values are then identified. In the later stage, the solutions are updated through the following equation:

$S_{u, v, w}^{\prime}=S_{u, v, w}+\operatorname{rand}_{1 u, v, w}\left(S_{b e s t, v, w}-S_{w o r s t, v, w}\right)$

where the original candidates are denoted by $S_{u, v, w}$, updated candidate solutions are denoted by $S_{u, v, w}^{\prime}$, best candidate solutions are represented by $S_{b e s t, v, w}$, and worst candidate solutions are represented by $S_{w o r s t, v, w}$. Then, a check is performed to determine if the new solution is better than the older solution and it replaces the old candidate solutions; otherwise, old candidate solutions are considered. This process is continued until the termination criteria are not met. The selected features are again evaluated through the fitness function and replace with the older features. Here, we obtained a feature vector of dimension $N \times 1104$ for feature vector 2 (ResNet features) and $N \times 522$ for feature vector 1 (DarkNet features).

Hybridization: Consider, $F_{k 1}$ denoting the selected optimal feature vector for ResNet101 through PSO of dimension $N \times 820$ and $F_{k 2}$ is the optimal feature vector for ResNet101 selected through the Rao-1 algorithm. Then, we find the correlation between features of $F_{k 1}$ and $F_{k 2}$. The features that have a strong correlation were selected. The Pearson correlation was used here to determine the correlation. The final vector for ResNet101 is denoted by $F_{\tilde{k}}$ of dimension $N \times 926$. Similarly, this process was performed on DarkNet53 features and obtained a feature vector of dimension $N \times 476$ denoted by $F_{\tilde{k} 1}$. Finally, both vectors were fused using the new PMDNR approach, which initially computes the standard deviation of the minimum length feature vector and performs padding according to the higher dimensional feature vector. Then, the Euclidean Distance (ED) between both vectors was calculated, and only those features with the minimum distance were considered. The ED is defined as follows:

$D(i, j)=\sqrt{\left(F_{\tilde{k} i}-F_{\tilde{k} 1 j}\right)^{2}}$

This formula was applied to all features, and we finally obtained a fused vector of dimension $N \times 1042$. However, we noted that this vector contained few redundant features and, therefore, compared all features through the Union method and considered the same value features only once in the final fused vector. Through this process, the final feature vector of dimension $N \times 840$ was obtained. These features were finally classified using an ELM classifier [46]. The predicted labeled results of ELM are illustrated in Fig. 5.

\section{Experimental Results and Analysis}

This section presents the experimental process. The classification results were computed for several classifiers using a 50:50 approach of training and testing. All classification results were computed using 10-fold validation. In the training process, we used Stochastic Gradient Descent for the learning of fine-tuned models. Moreover, the learning rate was 0.00001 , the mini-batch size was 64, and 100 epochs were used. The selected classifiers were ELM, Fine Tree, SVM, fine-KNN, and ensemble trees. The main classifier was ELM, which was used in this work for prediction, while the rest of the classifiers were considered for classification comparison. Each classifier's performance was calculated according to the following measures: sensitivity rate, precision rate, F1-score, accuracy, FNR, and testing time. Time was the most important measure in this work due to the optimization algorithms. All experiments are performed in MATLAB 2020b on a PC with 512 SSD storage, 16 GB RAM, and an 8 GB graphics card. 


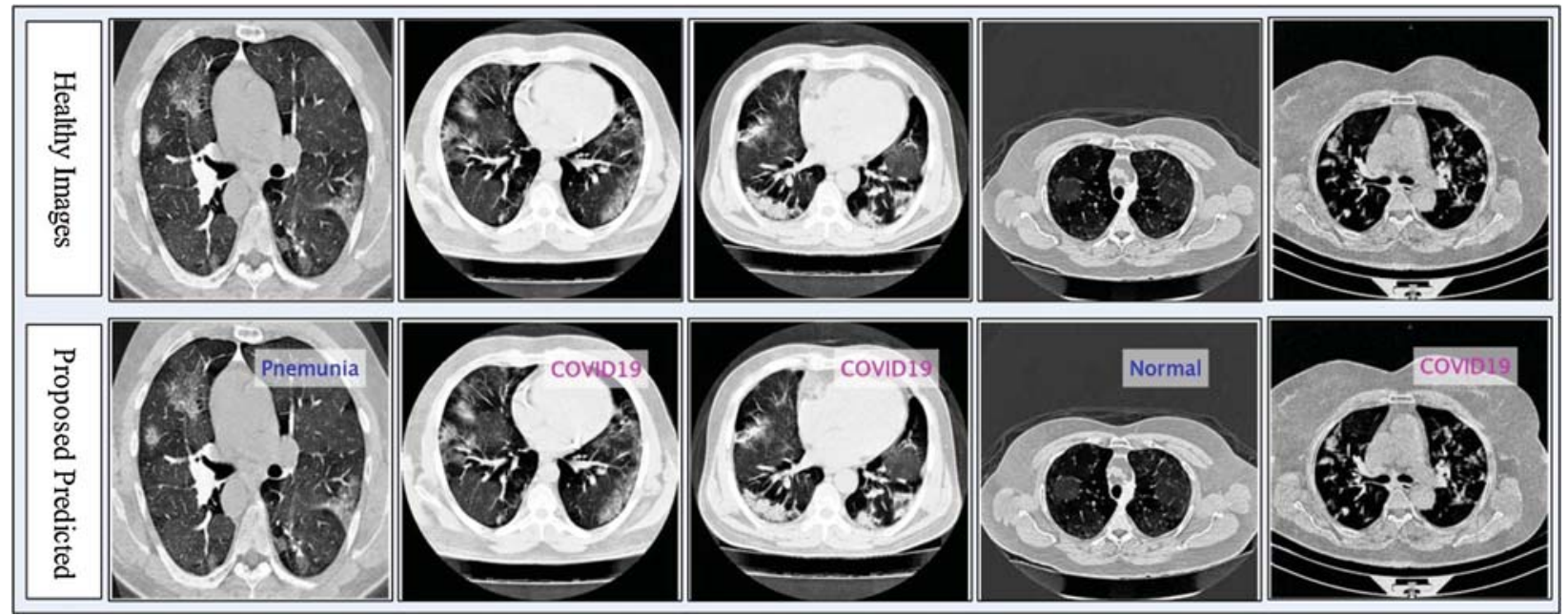

Figure 5: Proposed prediction results in terms of labeled data

\subsection{Numerical Results: Experiment 1}

The numerical results with accompanying evidence are presented in this section. The results were computed for each step. Tab. 1 presents the classification results after applying a hybrid optimization algorithm on fine-tuned ResNet50 model features. The results shown in this table were calculated for several classifiers using the selected performance measures. The greatest accuracy of this experiment was $92.7 \%$ for the ELM classifier. The computed accuracies for the remaining classifiers, i.e., Fine Tree to Softmax, were 86.2\%, 84.7\%, 83.2\%, 90.8\%, 90.7\%, 91.2\%, 91.4\%, and $92.1 \%$, respectively. These results indicated that the ELM classifier had the best performance. A number of other measures were also computed to support the performance of ELM; ELM showed sensitivity of $92.67 \%$, precision of $93 \%$, F1-Score of $92.83 \%$, and FNR of $7.33 \%$. To verify the sensitivity of ELM, a confusion matrix was added as shown in Fig. 6. This figure shows that the COVID-19 class correct prediction rate was $94 \%$, and the correct prediction rates for Pneumonia and Normal classes were $96 \%$ and $88 \%$, respectively. Thus, the prediction accuracy was best for the Pneumonia class followed by the COVID-19 class. The best time for this experiment was $25.706 \mathrm{~s}$, which was noted during the testing process of all $50 \%$ of images, indicating that the hybrid optimization algorithm minimized computational time in real-time testing.

\subsection{Numerical Results: Experiment 2}

Tab. 2 presents the classification results after applying the hybrid optimization algorithm on fine-tuned DarkNet53 model features. The results shown in this table were calculated for several classifiers using selected performance measures. The ELM classifier showed the highest accuracy of $92.40 \%$ in this experiment, which was $0.3 \%$ lower than in the experiment shown in Tab. 1 . The computed accuracies for the remaining classifiers, i.e., Fine Tree to Softmax, were 87.4\%, 88.2\%, $90.2 \%, 90.6 \%, 91.0 \%, 90.4 \%, 91.9 \%$, and $91.6 \%$, respectively. These results indicated that the ELM classifier had the best performance, and that the performance in this experiment was better than that in Tab. 1. A number of other measures were also computed to support the performance of ELM; ELM showed sensitivity of $92.40 \%$, precision of $92.7 \%$, F1-Score of $92.55 \%$, and FNR of $7.66 \%$. To verify the sensitivity of ELM, a confusion matrix was added as shown in Fig. 7. The time was also noted, and the best time for this experiment was 23.927 s showing that the 
accuracy of the ELM classifier using a hybrid optimized approach for DarkNet features executed faster than ResNet50 features. The performance of optimized DarkNet53 features was also better in comparison to Tab. 1.

Table 1: Proposed classification results after employing a hybrid optimization algorithm on finetuned ResNet50 deep feature vector

\begin{tabular}{lllllll}
\hline \multirow{2}{*}{ Classifier } & \multicolumn{5}{l}{ Evaluation Protocols } \\
\cline { 2 - 7 } & Sensitivity (\%) & Precision (\%) & F1-Score (\%) & Accuracy (\%) & FNR (\%) & Time (Sec) \\
\hline Fine Tree & 86.33 & 86.0 & 86.16 & 86.2 & 13.67 & 30.314 \\
GN Bayes & 84.66 & 85.3 & 84.98 & 84.7 & 15.34 & 49.532 \\
L-SVM & 83.33 & 83.3 & 83.31 & 83.2 & 16.67 & 22.965 \\
MG-SVM & 91.00 & 91.0 & 91.00 & 90.8 & 9.00 & 31.912 \\
C- KNN & 90.67 & 91.0 & 90.83 & 90.7 & 9.33 & 47.091 \\
W-KNN & 91.33 & 91.0 & 91.16 & 91.2 & 8.67 & $\mathbf{2 5 . 7 0 6}$ \\
EBT & 91.30 & 91.3 & 91.30 & 91.4 & 8.70 & 37.103 \\
Softmax & 92.00 & 92.0 & 92.00 & 92.1 & 8.00 & 24.014 \\
ELM & 92.67 & 93.0 & 92.83 & 92.7 & 7.33 & 30.775 \\
\hline
\end{tabular}

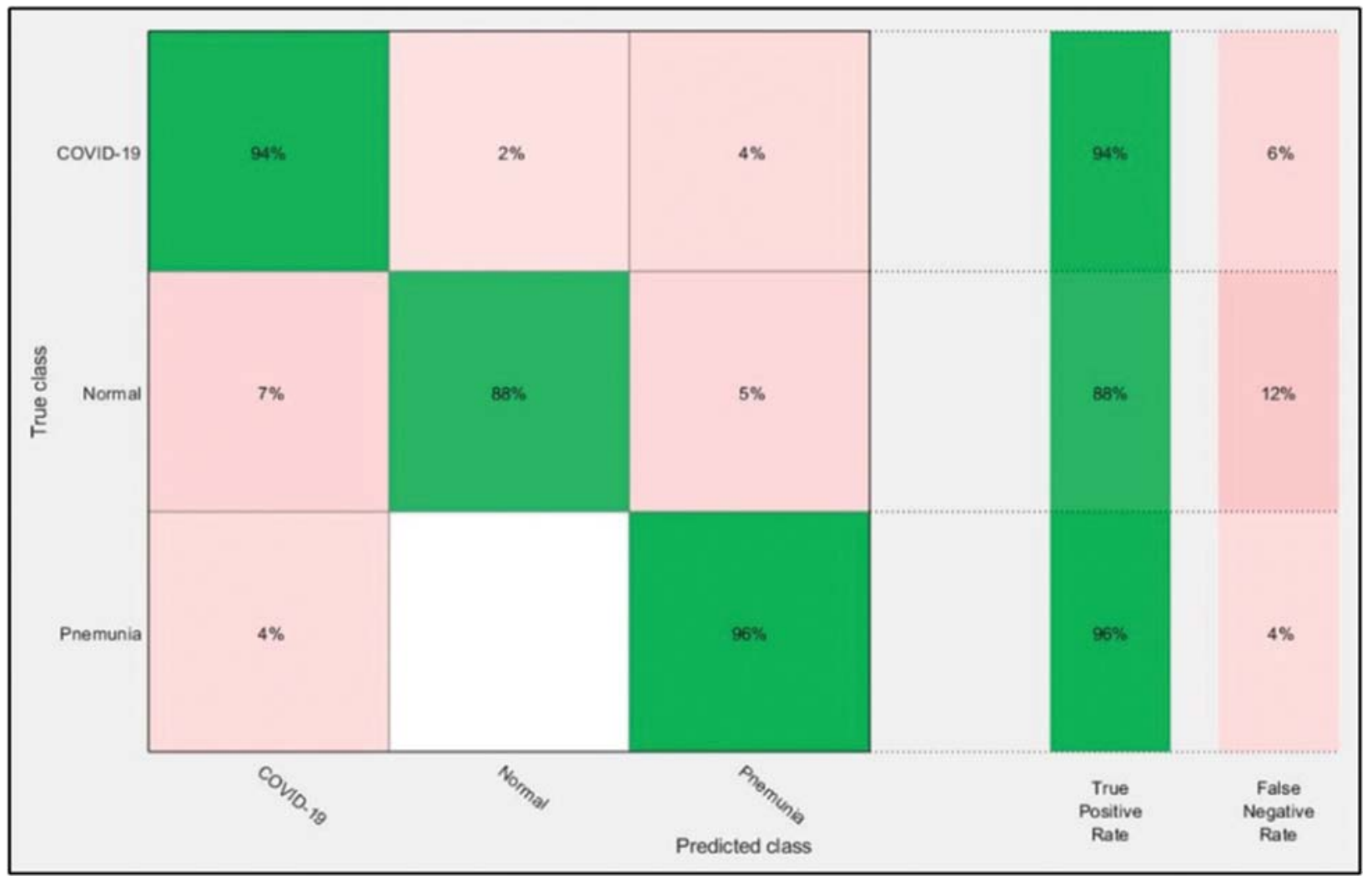

Figure 6: Confusion matrix of ELM for optimal ResNet50 deep learning features 
Table 2: Proposed classification results after employing a hybrid optimization algorithm on finetuned Darknet53 deep feature vector

\begin{tabular}{lllllll}
\hline \multirow{2}{*}{ Classifier } & \multicolumn{5}{l}{ Evaluation Protocols } \\
\cline { 2 - 7 } & Sensitivity (\%) & Precision (\%) & F1-Score (\%) & Accuracy (\%) & FNR (\%) & Time (Sec) \\
\hline Fine Tree & 87.47 & 87.5 & 87.48 & 87.4 & 12.53 & 36.652 \\
GN Bayes & 88.27 & 88.3 & 88.28 & 88.2 & 11.73 & 29.232 \\
L-SVM & 90.27 & 90.3 & 90.28 & 90.2 & 9.73 & 31.183 \\
MG-SVM & 90.60 & 90.6 & 90.60 & 90.6 & 9.40 & 35.348 \\
C- KNN & 91.07 & 91.3 & 91.18 & 91.0 & 8.93 & 34.172 \\
W-KNN & 90.40 & 90.5 & 90.45 & 90.4 & 9.60 & 32.772 \\
EBT & 92.00 & 92.0 & 92.00 & 91.9 & 8.0 & 43.216 \\
Softmax & 91.33 & 92.0 & 91.66 & 91.6 & 8.67 & $\mathbf{2 3 . 9 2 7}$ \\
ELM & 92.40 & 92.7 & 92.55 & 92.4 & 7.60 & 36.454 \\
\hline
\end{tabular}

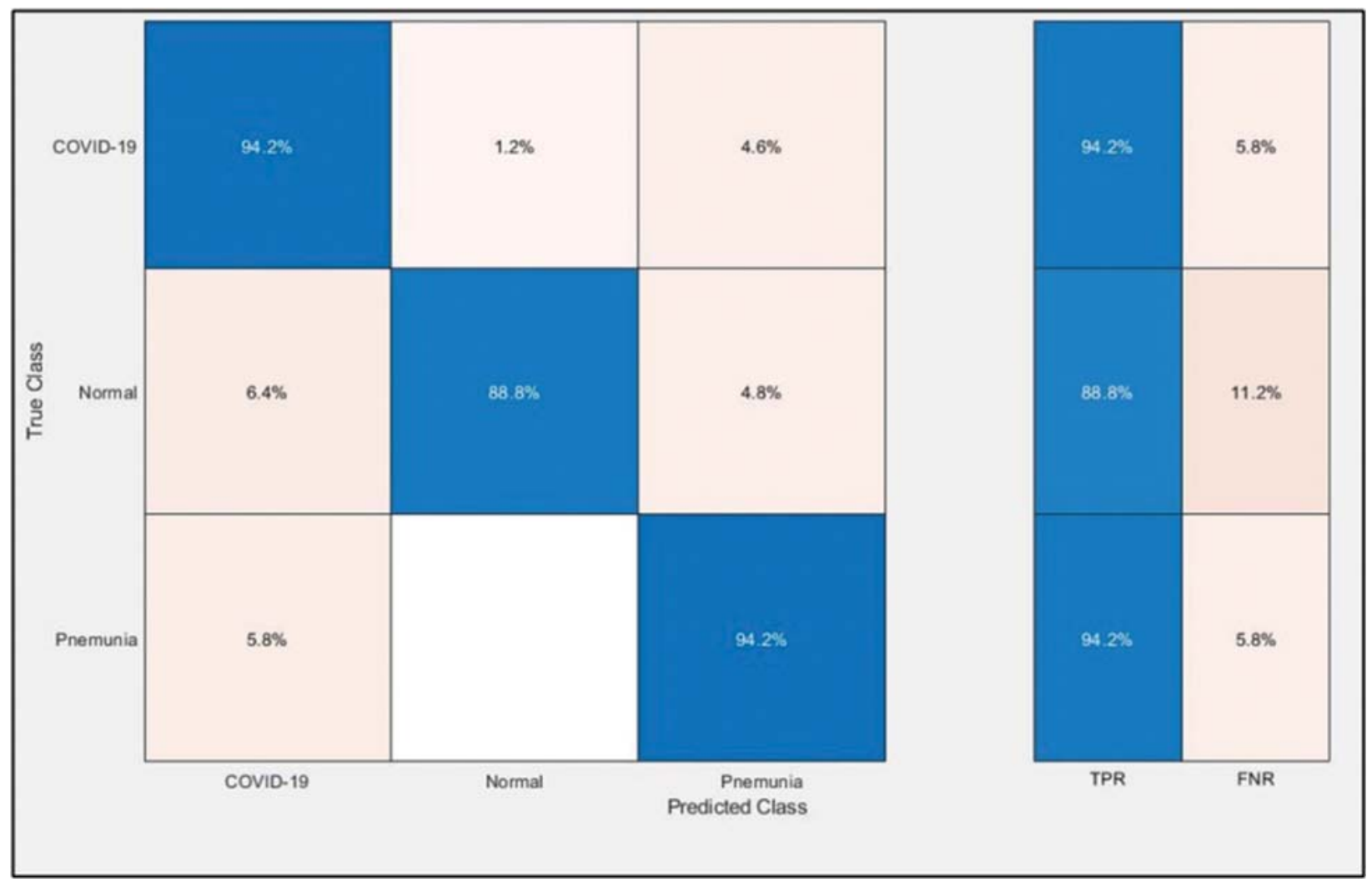

Figure 7: Confusion matrix of ELM for optimal DarkNet53 deep learning features 
Table 3: Proposed classification results after ensembles of deep learning models information fusion

\begin{tabular}{lllllll}
\hline Classifier & \multicolumn{5}{l}{ Evaluation Protocols } \\
\cline { 2 - 7 } & Sensitivity (\%) & Precision (\%) & F1-Score (\%) & Accuracy (\%) & FNR (\%) & Time (Sec) \\
\hline Fine Tree & 89.67 & 90.00 & 89.83 & 89.8 & 10.33 & 25.336 \\
GN Bayes & 94.13 & 94.33 & 94.23 & 94.5 & 5.87 & 21.325 \\
L-SVM & 93.13 & 93.67 & 93.40 & 93.6 & 6.87 & $\mathbf{1 5 . 6 0 9}$ \\
MG-SVM & 94.10 & 94.33 & 94.21 & 94.4 & 5.90 & 19.859 \\
C- KNN & 94.00 & 94.00 & 94.00 & 94.1 & 6.00 & 26.194 \\
W-KNN & 93.03 & 93.33 & 93.18 & 93.4 & 6.97 & 17.312 \\
EBT & 93.00 & 93.33 & 93.16 & 93.0 & 7.00 & 23.010 \\
Softmax & 94.33 & 94.60 & 94.46 & 94.7 & 5.67 & 22.699 \\
ELM & 95.33 & 95.67 & 95.50 & 95.6 & 4.67 & 20.003
\end{tabular}

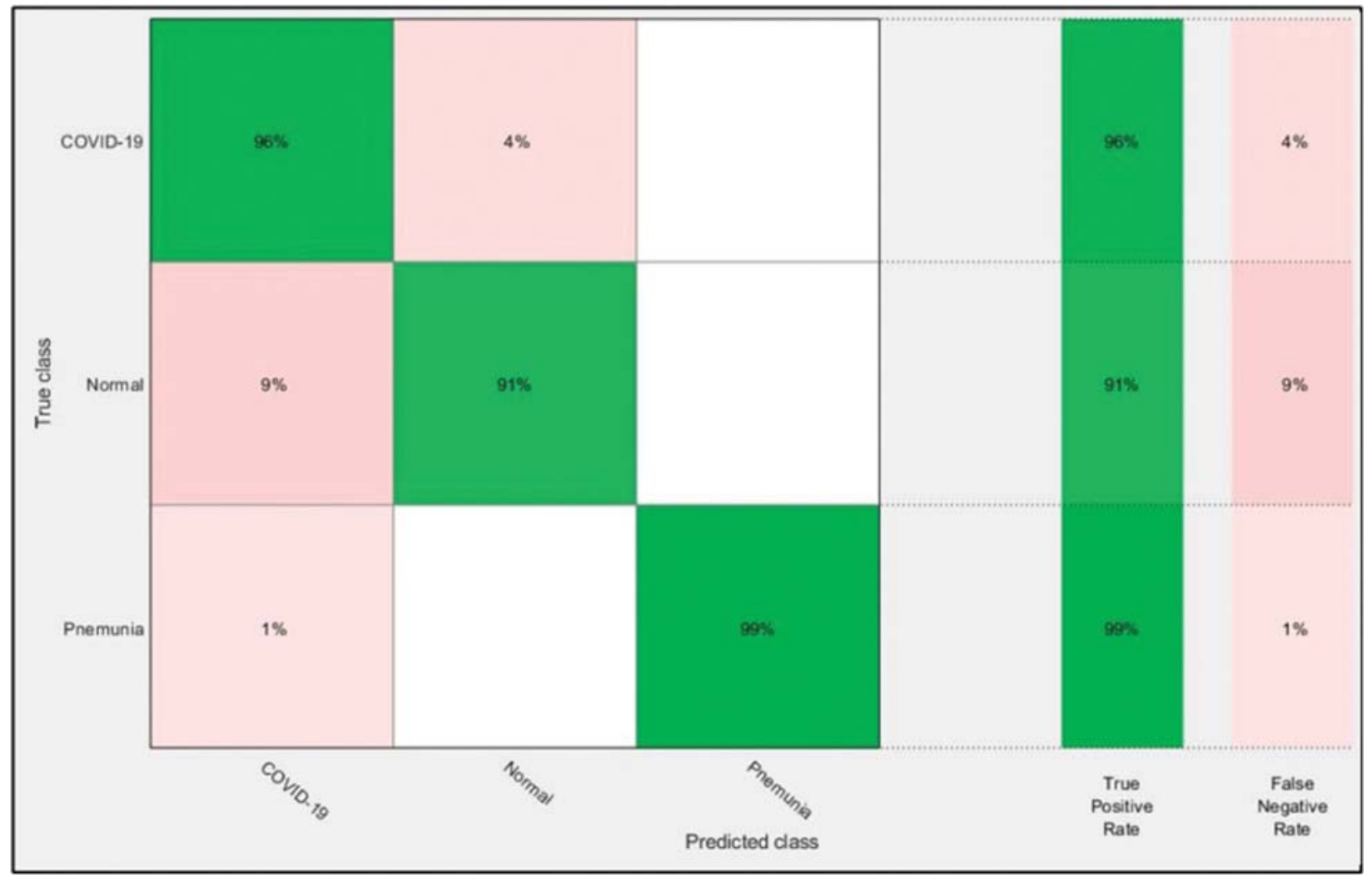

Figure 8: Confusion matrix of ELM after ensembles of deep learning models information fusion 


\subsection{Numerical Results: Experiment 3}

The optimal features of both models were fused using the proposed PMDNR approach, and the results are presented in Tab. 3. The results shown in this table indicated that the highest accuracy achieved was $95.6 \%$ for the ELM classifier. The other measures were sensitivity of $95.33 \%$, precision of $95.67 \%, \mathrm{~F} 1-$ Score of $95.50 \%$, and FNR of $4.67 \%$. The noted computational time of ELM was $20.003 \mathrm{~s}$, which was less than in Tabs. 1 and 2. In addition, the accuracy of ELM after fusion was improved compared to Tabs. 1 and 2. Fig. 8 shows a confusion matrix, which can be used to verify the sensitivity of ELM. As shown in this figure, the correct prediction rates for Pneumonia and COVID-19 images were 99\% and 96\%, respectively, representing significant improvements compared to Figs. 6 and 7.

Finally, we discuss the overall experimental process of our proposed framework. Fig. 9 shows the proposed framework accuracy with individual steps. Initially, the results were computed using original ResNet50 ( $O_{-} R_{2} s_{-} F$ ) features and achieved an accuracy of $87.86 \%$. For the original DarkNet53 ( $\left.O \_D r k \_F\right)$ features, the results accuracy was $85.62 \%$. The results of optimal ResNet50 features were $92.7 \%$, which were improved compared to $O \_R e s \_F$. Similarly, the results were improved after employing optimal DarkNet53 (OP_Drk_F) and reached 92.4\%. The results are illustrated for a proposed framework of accuracy of $95.6 \%$. The proposed framework results demonstrated the significance for classification of COVID-19 on CT images. Similarly, the F1Score-based comparison was also conducted as shown in Fig. 10. This figure shows that the proposed framework achieved better results on the ELM classifier. A time-based comparison was also conducted shown in Fig. 11, which indicated that the ensembles of deep features executed faster, compared to the ResNet and DarkNet features.

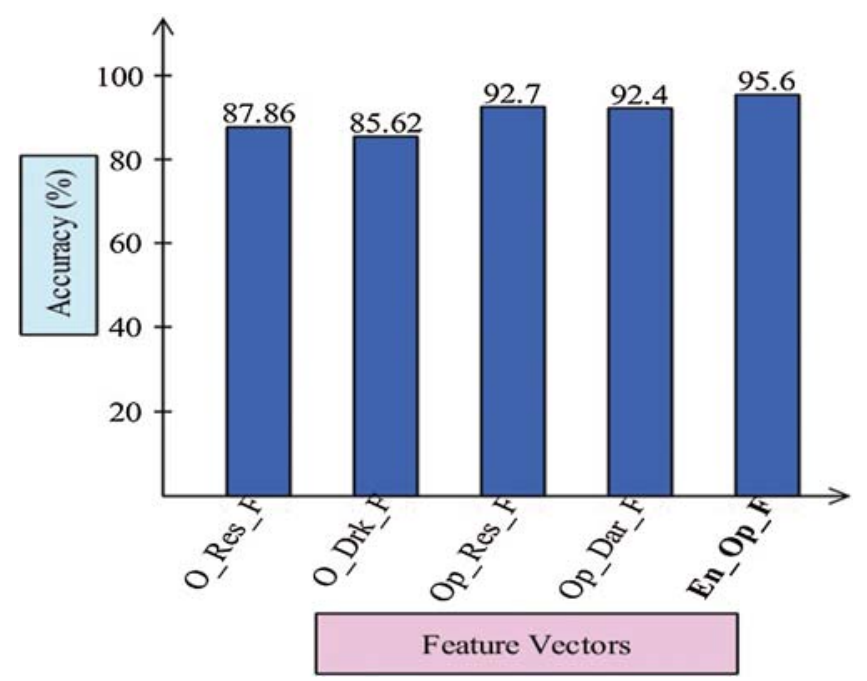

Figure 9: Proposed ensemble method accuracy comparison with individual steps 


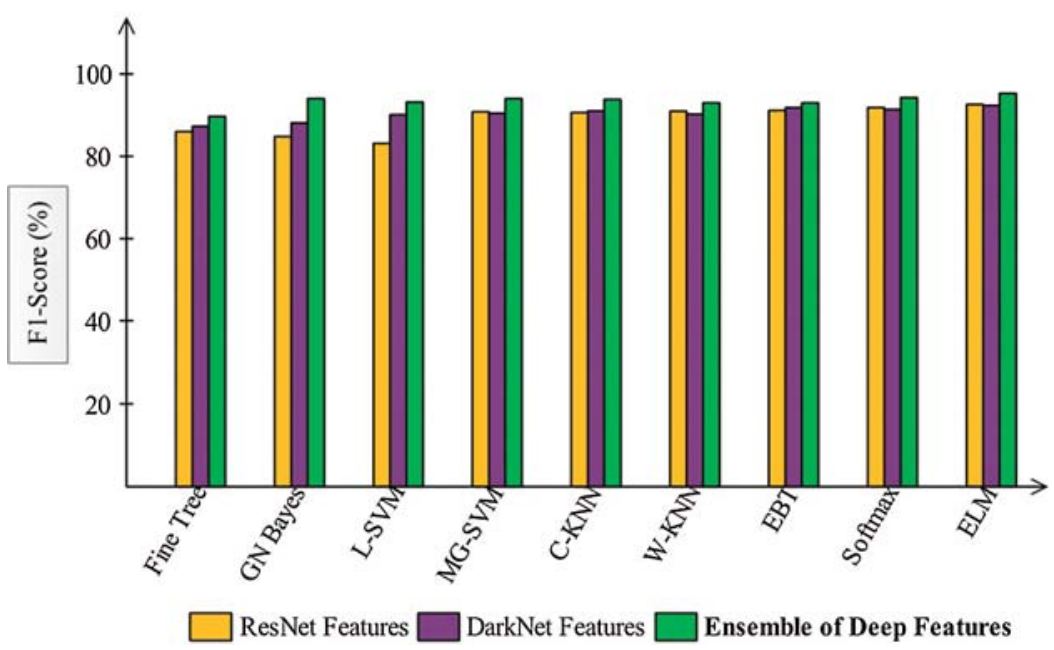

Figure 10: F1-Score based comparison among each step for the selected classifiers

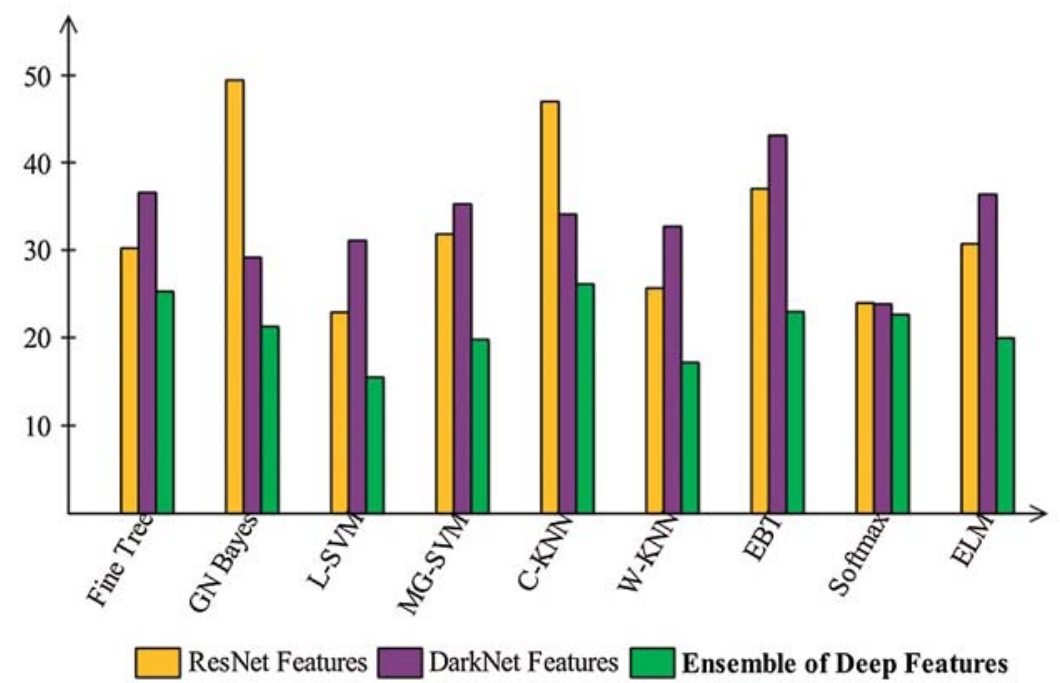

Figure 11: Time plot to compare the performance of each step

\section{Conclusion}

To classify COVID-19 CT images, a set of optimal deep learning features based on a computerized framework was presented. This work allowed the selection of the most optimal features using a hybrid algorithm and the fusion of features through the proposed PMDNR approach. Finally, the fused features were classified using an ELM classifier. The results were computed on the prepared database and showed $95.6 \%$ accuracy. The results indicated that the proposed method works better with the ELM classifier. Pre-processing of the original images improved the visibility of infected and curative CT images from the results. Use of improved contrast images often allowed the extraction of more useful deep learning features. In addition, optimizing features improved the accuracy of the system and minimized the number of predictors required. 
With fewer predictors, the procedure was completed quickly, which is helpful for improving the performance of the real-time system. We also concluded that the fusion of features using the proposed approach further impaired accuracy. In future, the features will be extracted through two or three stream networks and fused for more accurate classification accuracy.

Funding Statement: This research was supported by X-mind Corps program of National Research Foundation of Korea (NRF) funded by the Ministry of Science, ICT (No. 2019H1D8A1105622) and the Soonchunhyang University Research Fund.

Conflicts of Interest: The authors declare that they have no conflicts of interest to report regarding the present study.

\section{References}

[1] S. B. Stoecklin, P. Rolland, Y. Silue, A. Mailles, C. Campese et al. "First cases of coronavirus disease 2019 (COVID-19) in France: Surveillance, investigations and control measures," Eurosurveillance, vol. 25, no. 3, pp. 2000094, 2020.

[2] N. Zhu, D. Zhang, W. Wang, X. Li, B. Yang et al. "A novel coronavirus from patients with pneumonia in China," New England Journal of Medicine, vol. 4, no. 7, pp. 1-4, 2020.

[3] T. Singhal, "A review of coronavirus disease-2019 (COVID-19)," Indian Journal of Pediatrics, vol. 6, no. 2, pp. 1-6, 2020.

[4] C. Wang, P. W. Horby, F. G. Hayden and G. F. Gao, "A novel coronavirus outbreak of global health concern," Lancet, vol. 395, no. 2, pp. 470-473, 2020.

[5] T. Ai, Z. Yang, H. Hou, C. Zhan, C. Chen et al. "Correlation of chest CT and RT-pCR testing in coronavirus disease 2019 (COVID-19) in China: A report of 1014 cases," Radiology, vol. 7, no. 11, pp. 200642, 2020.

[6] F. M. Salman, S. S. Abu-Naser, E. Alajrami, B. S. Abu-Nasser and B. A. Alashqar, "Covid-19 detection using artificial intelligence," International Journal of Academic Engineering Research, vol. 4, no. 3, pp. 1825, 2020.

[7] A. Mittal, D. Kumar, M. Mittal, T. Saba, I. Abunadi et al. "Detecting pneumonia using convolutions and dynamic capsule routing for chest X-ray images," Sensors, vol. 20, no. 1, pp. 1068, 2020.

[8] M. A. Khan, M. S. Sarfraz, M. Alhaisoni, A. A. Albesher, S. Wang et al. "Stomachnet: Optimal deep learning features fusion for stomach abnormalities classification," IEEE Access, vol. 8, no. 11, pp. 197969-197981, 2020.

[9] A. Rehman, M. Khan, T. Saba, Z. Mehmood, U. Tariq et al. "Microscopic brain tumor detection and classification using 3D CNN and feature selection architecture," Microscopy Research and Technique, vol. 5 , no. 3 , pp. $1-21,2020$.

[10] S. A. C. Bukhari and R. S. Nayak, "Developed newton-raphson based deep features selection framework for skin lesion recognition," Pattern Recognition Letters, vol. 129, no. 2, pp. 293-303, 2020.

[11] M. Alhaisoni, R. Damaševičius and R. Scherer, "Multimodal brain tumor classification using deep learning and robust feature selection: A machine learning application for radiologists," Diagnostics, vol. 10, pp. 565, 2020.

[12] M. A. Khan, S. Kadry, M. Alhaisoni, Y. Nam, Y. Zhang et al. "Computer-aided gastrointestinal diseases analysis from wireless capsule endoscopy: A framework of best features selection," IEEE Access, vol. 8, no. 7, pp. 132850-132859, 2020.

[13] S. Rubab, A. Kashif, M. I. Sharif and N. Muhammad, "Lungs cancer classification from CT images: An integrated design of contrast based classical features fusion and selection," Pattern Recognition Letters, vol. 129, pp. 77-85, 2020.

[14] S. Zahoor, I. U. Lali, M. A. Khan, K. Javed and W. Mehmood, "Breast cancer detection and classification using traditional computer vision techniques: A comprehensive review," Current Medical Imaging, vol. 7, no. 17, pp. 1-31, 2020. 
[15] H. M. J. Lodhi, M. Nazir, K. Javed and M. Qasim, "Automated design for recognition of blood cells diseases from hematopathology using classical features selection and ELM," Microscopy Research and Technique, vol. 7, no. 5, pp. 1-19, 2020.

[16] G. R. Shinde, A. B. Kalamkar, P. N. Mahalle, N. Dey, J. Chaki et al. "Forecasting models for coronavirus disease (COVID-19): A survey of the state-of-the-art," SN Computer Science, vol. 1, no. 3, pp. 1-15, 2020.

[17] S.-H. Wang, X. Wu, Y.-D. Zhang, C. Tang and X. Zhang, "Diagnosis of COVID-19 by wavelet renyi entropy and three-segment biogeography-based optimization," International Journal of Computational Intelligence Systems, vol. 13, pp. 1332-1344, 2020.

[18] Y.-D. Zhang, S. C. Satapathy, S. Liu and G.-R. Li, "A five-layer deep convolutional neural network with stochastic pooling for chest CT-based COVID-19 diagnosis," Machine Vision and Applications, vol. 32, no. 2, pp. 1-13, 2020.

[19] Y.-D. Zhang, S. C. Satapathy, L.-Y. Zhu, J. M. Górriz and S.-H. Wang, "A seven-layer convolutional neural network for chest CT based COVID-19 diagnosis using stochastic pooling," IEEE Sensors Journal, vol. 1, no. 13, pp. 1-9, 2020.

[20] X. Yu, S. Lu, L. Guo, S.-H. Wang and Y.-D. Zhang, "Resgnet-c: A graph convolutional neural network for detection of COVID-19," Neurocomputing, vol. 3, pp. 1-18, 2020.

[21] S.-H. Wang, D. R. Nayak, D. S. Guttery, X. Zhang, and Y.-D. Zhang, "COVID-19 classification by CCSHNet with deep fusion using transfer learning and discriminant correlation analysis," Information Fusion, vol. 68, pp. 131-148, 2020.

[22] M. Alazab, A. Awajan, A. Mesleh, A. Abraham, V. Jatana et al. "COVID-19 prediction and detection using deep learning," International Journal of Computer Information Systems and Industrial Management Applications, vol. 12, no. 26, pp. 168-181, 2020.

[23] I. D. Apostolopoulos and T. A. Mpesiana, "Covid-19: Automatic detection from x-ray images utilizing transfer learning with convolutional neural networks," Physical and Engineering Sciences in Medicine, vol. 6, no. 2, pp. 1, 2020.

[24] T. Ozturk, M. Talo, E. A. Yildirim, U. B. Baloglu, O. Yildirim et al. "Automated detection of COVID19 cases using deep neural networks with X-ray images," Computers in Biology and Medicine, vol. 23, pp. 103792, 2020.

[25] A. M. Ismael and A. Şengür, "Deep learning approaches for COVID-19 detection based on chest X-ray images," Expert Systems with Applications, vol. 164, pp. 114054, 2020.

[26] H. Panwar, P. Gupta, M. K. Siddiqui, R. Morales-Menendez, P. Bhardwaj et al. "A deep learning and grad-cAM based color visualization approach for fast detection of COVID-19 cases using chest X-ray and CT-scan images chaos," Solitons \& Fractals, vol. 140, pp. 110190, 2020.

[27] E. Hussain, M. Hasan, M. A. Rahman, I. Lee, T. Tamanna et al. "Corodet: A deep learning based classification for COVID-19 detection using chest X-ray images chaos," Solitons \& Fractals, vol. 11, pp. 110495, 2020.

[28] M. Z. Islam, M. M. Islam and A. Asraf, "A combined deep CNN-1STM network for the detection of novel coronavirus (COVID-19) using X-ray images," Informatics in Medicine Unlocked, vol. 20, no. 6, pp. 100412, 2020.

[29] S. Toraman, T. B. Alakus and I. Turkoglu, "Convolutional capsnet: A novel artificial neural network approach to detect COVID-19 disease from X-ray images using capsule networks chaos," Solitons \& Fractals, vol. 140, pp. 110122, 2020.

[30] K. Gao, J. Su, Z. Jiang, L.-L. Zeng, Z. Feng et al. "Dual-branch combination network (DCN): Towards accurate diagnosis and lesion segmentation of COVID-19 using CT images," Medical Image Analysis, vol. 67, no. 1, pp. 101836, 2020.

[31] M. J. Horry, S. Chakraborty, M. Paul, A. Ulhaq, B. Pradhan et al. "COVID-19 detection through transfer learning using multimodal imaging data," IEEE Access, vol. 8, pp. 149808-149824, 2020.

[32] M. Arora, L. M. Goyal, N. Chintalapudi and M. Mittal, "Factors affecting digital education during COVID-19: a statistical modeling approach," in 2020 5th Int. Conf. on Computing, Communication and Security (ICCCS), Patna, India, pp. 1-5, 2020. 
[33] M. Mittal, G. Battineni, L. M. Goyal, B. Chhetri, S. V. Oberoi et al. "Cloud-based framework to mitigate the impact of COVID-19 on seafarers' mental health," International Maritime Health, vol. 71, pp. 213-214, 2020.

[34] J. K. Sethi and M. Mittal, "Monitoring the impact of air quality on the COVID-19 fatalities in Delhi, India: Using machine learning techniques," Disaster Medicine and Public Health Preparedness, vol. 8, pp. 1-8, 2020.

[35] A. Liaqat, M. Sharif, M. Mittal and T. Saba, "Gastric tract infections detection and classification from wireless capsule endoscopy using computer vision techniques: A review," Current Medical Imaging, vol. 22, pp. 1-31, 2020.

[36] M. Mittal, L. M. Goyal, S. Kaur, I. Kaur, A. Verma et al., "Deep learning based enhanced tumor segmentation approach for MR brain images," Applied Soft Computing, vol. 78, pp. 346-354, 2019.

[37] M. Rashid, M. A. Khan, M. Alhaisoni, S.-H. Wang, S. R. Naqvi et al. "A sustainable deep learning framework for object recognition using multi-layers deep features fusion and selection," Sustainability, vol. 12, pp. 5037, 2020.

[38] A. Farhadi and J. Redmon, "Yolov3: An incremental improvement," Computer Vision and Pattern Recognition, vol. 2, no. 7, pp. 1-6, 2018.

[39] J. Redmon and A. Farhadi, "YOLO9000: Better, faster, stronger," in Proc. of the IEEE Conf. on Computer Vision and Pattern Recognition, Honolulu, Hawaii, pp. 7263-7271, 2017.

[40] K. He, X. Zhang, S. Ren and J. Sun, "Deep residual learning for image recognition," in Proc. of the IEEE Conf. on Computer Vision and Pattern Recognition, Las Vegas, Nevada, pp. 770-778, 2016.

[41] J. Deng, W. Dong, R. Socher, L.-J. Li, K. Li et al. "Imagenet: A large-scale hierarchical image database," in 2009 IEEE Conf. on Computer Vision and Pattern Recognition, Miami, FL, USA, pp. 248255, 2009.

[42] N. Naheed, M. Shaheen, S. A. Khan, M. Alawairdhi and M. A. Khan, "Importance of features selection, attributes selection, challenges and future directions for medical imaging data: A review," Computer Modeling in Engineering \& Sciences, vol. 125, no. 3, pp. 314-344, 2020.

[43] R. V. Rao and R. Pawar, "Constrained design optimization of selected mechanical system components using rao algorithms," Applied Soft Computing, vol. 89, no. 4, pp. 106141, 2020.

[44] S. Yadav, A. Ekbal and S. Saha, "Feature selection for entity extraction from multiple biomedical corpora: A PSO-based approach," Soft Computing, vol. 22, no. 11, pp. 6881-6904, 2018.

[45] M. Amoozegar and B. Minaei-Bidgoli, "Optimizing multi-objective PSO based feature selection method using a feature elitism mechanism," Expert Systems with Applications, vol. 113, no. 26, pp. 499-514, 2018.

[46] G.-B. Huang, X. Ding and H. Zhou, "Optimization method based extreme learning machine for classification," Neurocomputing, vol. 74, no. 2, pp. 155-163, 2010. 